\title{
ODAP: A stand-alone program for observational data acquisition
}

\author{
WILLIAM P. HETRICK, ROBERT C. ISENHART, DEREK V. TAYLOR, \\ and CURT A. SANDMAN \\ State Developmental Research Institute, Fairview, Costa Mesa, California
}

\begin{abstract}
A software program written to collect real-time, observational data is described. The flexible program allows customized behavior codes and observational durations and simultaneously records both timed and counted events. The data are collected by means of single keystrokes, automatically stored to disk with 100th of a second resolution and summarized for each observational session. The program's database files are dBASE III PLUS compatible and may be browsed, edited, or converted to ASCII files from the program's main menu. Field testing demonstrated the efficiency and interobserver reliability of the program (for frequency, $r=0.81$; for durational behaviors, $r \mathrm{~s}=0.89$ and 0.96 ). The software operates on IBM XT/AT/PS $2 \mathrm{~s}$ and most clones with PC/MS DOS version 2.0 or greater.
\end{abstract}

Observational research is often impractical because of imprecise and burdensome procedures. Traditional methods of mechanical recording typically require observers to perform several tasks, such as monitoring time and recalling behavior repertoires, categories, or codes, while recording behavior systematically with paper and pencil. Even in controlled laboratory settings, observational data acquisition can be difficult. When collecting observational data from human subjects, particularly patients, the reliability of the data may be compromised by the combination of procedural requirements and the behavioral diversity of the subjects. These difficulties often lead researchers to compromise their data collection requirements in order to obtain reliable data.

Computer-assisted observational methods have greatly reduced the task of collecting and transforming raw behavioral data into meaningful measures (Flowers \& Leger, 1982). In the early to mid 1970 s, electronic data acquisition systems began to be reported in the literature (see discussions and reviews by Fitzpatrick, 1977; Sackett, Stephenson, \& Ruppenthal, 1973; Sidowski, 1977). These electronically primitive machines (compared with current microcomputer technology) with limited internal memory (RAM and ROM) and/or archaic storage mediums (e.g., computer tape/cards and audio cassette tapes) were followed by early model programmable microcomputers with increased memory capacity and internal real-time clocks (e.g., Crossman, Williams, \& Chambers, 1978; Koontz,

We gratefully acknowledge the assistance of Tom Ortiz and Diane Rush in field testing and data collection. Preparation of this manuscript was supported in part by National Institute of Mental Health Grant R01 MH41446. The authors are also affiliated with the Department of Psychiatry and Human Behavior, University of California, Irvine. Address correspondence to William Hetrick, Research Box 5A, 2501 Harbor Blvd., Costa Mesa, CA 92626.
1982; Lifshitz, O’Keeffe, Lee, \& Avery, 1985). Concurrently, hand-held, or pocket, microcomputers (e.g., Radio Shack PC-2, Sharp PC-1500) were becoming available and used for observational data acquisition (Elias, 1984). Both the early model microcomputers and the hand-held computers had limited storage space and typically required transfer of data to a main frame or larger capacity computer for summary and analysis. Most of the systems described above, however, are now outdated by recent technology (both hardware and software) and may be difficult to secure for future use.

The most recent generation of electronic/computeraided event recorders include (1) versatile hand-held computers, (2) an instrument using current bar code technology, and (3) software programs for personal and laptop computers which collect real-time observational data.

A data acquisition computer named "The Assistant" (Human Technologies, Inc., 300 3rd Ave. North, St. Petersburg, FL, 33701) exemplifies the features of programmable, multipurpose hand-held computers. The Assistant allows tracking of up to 10 counted (frequency) behaviors or four timed (duration) behaviors, storing as many as 800 total events and includes a programmable timer. The inability to record both timed and counted behaviors simultaneously is restricting. Data are entered on a numeric key pad on the face of the hand-held computer with each of the 10 keys corresponding to a behavior or event. This feature facilitates the rate of data entry since only one keypress is necessary to record occurrence or onset/ offset of events. The resolution is programmable from $2 \mathrm{sec}$ to $1 \mathrm{~h}$. As with all hand-held computers, limited data storage capacity is the cost of portability. The Assistant (\$325) outputs data to a printer or data file for further manipulation; also, an optional data analysis software package is available (\$229).

A recently developed and novel computer-aided method for observational data collection includes the use of bar 
code technology (Eiler, Nelson, Jensen, \& Johnson, 1989). With this method,

behavior observations are recorded from multiple bar code symbols printed on a one-page entry form, or data menu. $A$ unique bar code symbol is assigned to each possible observation, and the research staff scan the corresponding symbol on the data menu to record the event and automatically log the observational time. (p. 53)

Advantages of the bar code scanner include portability, accuracy, and reduced observer training time. Potential disadvantages include the 15-sec resolution of the scanner's internal clock, a 2-3 sec lag time required to align the scanner's read head with the desired bar code, apparent inability to record event duration, and the necessity of transferring data from the scanner to a host computer. The data acquisition rate of the highest capacity scanner, as stated in the following ratio, might be restricting: "one observation every $15 \mathrm{sec}$ for an 8 -h period" (Eiler et al., 1989 , p. 55). Cost of a scanner and data transfer device ranges from $\$ 347$ to $\$ 397$, depending on memory capacity of the scanner.

EVENTLOG, a real-time event recording software program, represents a third type of observational data acquisition instrument (CONDUIT, University of lowa, Oakdale Campus, Iowa City, IA, 52242). Unlike the specialized hand-held computer and bar code scanner described above, which require a host computer for data storage and anal$y s i s$, in addition to specialized hardware for data entry, software programs such as EVENTLOG operate in common, industry standard computer systems (i.e., IBM PC/ XT/AT/PS-2 and most compatibles). With EVENTLOG, keys on the standard keyboard are configured to correspond with the events or behaviors of interest. A key is depressed for the duration of the corresponding event with the keypress, key release, and duration recorded with millisecond accuracy. EVENTLOG maintains accuracy when several keys are pressed simultaneously. Additional features include programmable visual and/or auditory timers to indicate time intervals, the ability to make notes that are recorded in the data file, and compatibility of the output file with major statistical packages. EVENTLOG is priced at $\$ 125$.

In the present paper, we introduce a versatile, standalone Observational Data Acquisition Program (ODAP) that allows on-line recording of both frequency and temporal features of behavior. The program is tailored to facilitate reliable, real-time recording of fast-changing behaviors. Although EVENTLOG compares favorably to ODAP and has the advantage of being able to record up to $40+$ events, ODAP appears better suited for observational conditions under which a limited number ( 10 or less) of highly variable behaviors/events must be recorded rapidly. ODAP's data acquisition procedures are programmable to achieve maximum automation; data are managed and stored in DBASE III compatible database files that are easily converted, from within ODAP's menus, to ASCII files for compatibility with major statistical packages. The data are output to two files, one of which is in a raw, data stream format and the other a summary file that is appended after each observation session with total frequencies and durations of target behaviors. Also, browsing and editing capabilities are incorporated in ODAP. Programmability renders ODAP suitable for various observational methods such as event recording and focal-individual sampling providing frequency, duration, and rate of occurrence information as well as the ability to conduct time budget estimates and behavioral sequence analysis (Altmann, 1974; Cone \& Foster, 1982; Lehner, 1979).

ODAP was tested using the focal-individual sampling method (Altmann, 1974). With this method, a subject " is observed for a predetermined period of time, during which all occurrences of specified behaviors and the time at which they take place are recorded" (Koontz, 1982, p. 431). Although the program was designed, tested, and used for human observational research of self-injurious behavior in the developmentally delayed, it has diverse applications. The program may be executed from a laptop computer in the field, from a stationary system in the lab, or adjacent to video playback equipment. The latter method was used by our researchers because videotape analysis was preferred.

\section{Program Initialization}

The program begins with an initialization dialogue in which the user is prompted for a subject name (alphanumeric characters are allowed), followed by date and time variables. Three additional user-defined variables for the categorization of observational sessions may be defined and subsequently modified through the "Modify Labels" option on the main menu. One of the variables, for example, could label each session for a specific experimental condition. A total of seven variables may be used to define each observational session: subject name, date, time, up to three user-defined labels, and the observational session number.

ODAP is capable of handling an unlimited number of observational sessions, with disk space the only limitation. During the initialization dialogue, the observer is prompted for the total number of observational sessions to collect during the current data collection procedure (range $=1$ to 999). Next, a prompt appears for the maximum duration of the sessions to be collected (range $=5$ to $14,000 \mathrm{sec}$ ). If variable interval durations are desired, then the maximum duration for any given session within a group can be programmed at the menu and a manual stop invoked by the user at the end of each session; otherwise, the program terminates the data acquisition mode at the desired duration.

Up to 10 behavior categories are allowed. These might represent, for example, the behavior code, or behavioral repertoire, of a focal subject or the target behaviors of a research population (see Hawkins, 1982, for a discussion of and procedures for developing behavior codes). Each time the program is executed, a search for the subject is made in a database file containing subject names and corresponding behavior codes. If a match is not found, then a prompt to define a behavior code is issued. As many 
as seven counted and three timed behaviors may be defined and recorded simultaneously. Once entered, the code is stored to disk for subsequent use, then these behavioral descriptors serve as labels for keys 0 to 9 on a numeric key-pad template displayed on the computer monitor (see Figure 1).

\section{Data Input}

After the initialization procedures, the observer issues a command to place the program in the "scoring" mode and to begin collecting data. The real-time clock, with 100 th of a second accuracy, is started, and the computer is ready to record keystrokes and their times of occurrence. If the program is used to score videotaped behavior, then the tape can be started simultaneously. When a behavior occurs in one of the preassigned categories, the "scorer" presses the appropriate key. This causes a marker to appear in the corresponding key's box (see Figure 1) and remain until another key is pressed. For timed behaviors, an "ON" marker appears in the corresponding box when a key is pressed and is removed after the second press, indicating the end of that behavior. The user may continue this scoring procedure, pressing appropriate keys once for frequency-type behaviors and at the onset and termination of duration-type behaviors. If the user enters a wrong numeric keystroke for counted behaviors, the minus key ( - ) may be pressed to erase it.

Throughout the data entry process, feedback in the form of screen markers, informational displays, and prompts alert the observer to special conditions or problems as well as the ongoing operational status. All inappropriate keystrokes invoke a momentary "invalid key" error mes- sage. Also, the program may be suspended/paused during operation and then either aborted and reset or resumed for continuation.

The scoring mode terminates automatically when the preassigned session duration expires or manually when the user types ' $P$ " to pause. In either case, the program displays a menu offering the following options: (1) abort the current session and restart, (2) increment session or quit, or (3) resume scoring.

The user has the option of defining up to 10 rating prompts, which appear at the end of scoring each session. For our use of the program to study self-injurious behavior, the observer was prompted for ratings of behavior severity and general physical activity during the preceding session. The prompts may be defined, edited, or deleted by selecting the "Modify Behavior Ratings" option from the main menu.

After responding to rating prompts, if any are defined, a message appears asking the user to type " $Q$ " to quit or " $\mathrm{C}$ " to continue with scoring the next session.

\section{Data Storage and Summarization}

The often tedious task of transforming the raw behavioral record into meaningful measures is ameliorated by ODAP. Data are stored directly to disk in dBASE III PLUS compatible database files. Using the "Output ASCII File" option from the menu, database files are converted to "free format" ASCI files (i.e., fields are separated by one blank space), which are compatible with most major statistical packages (e.g., BMDP and SPSS). The "Browse/Edit Database Files" menu option allows browsing, editing, and deleting of data. Owners of dBASE III

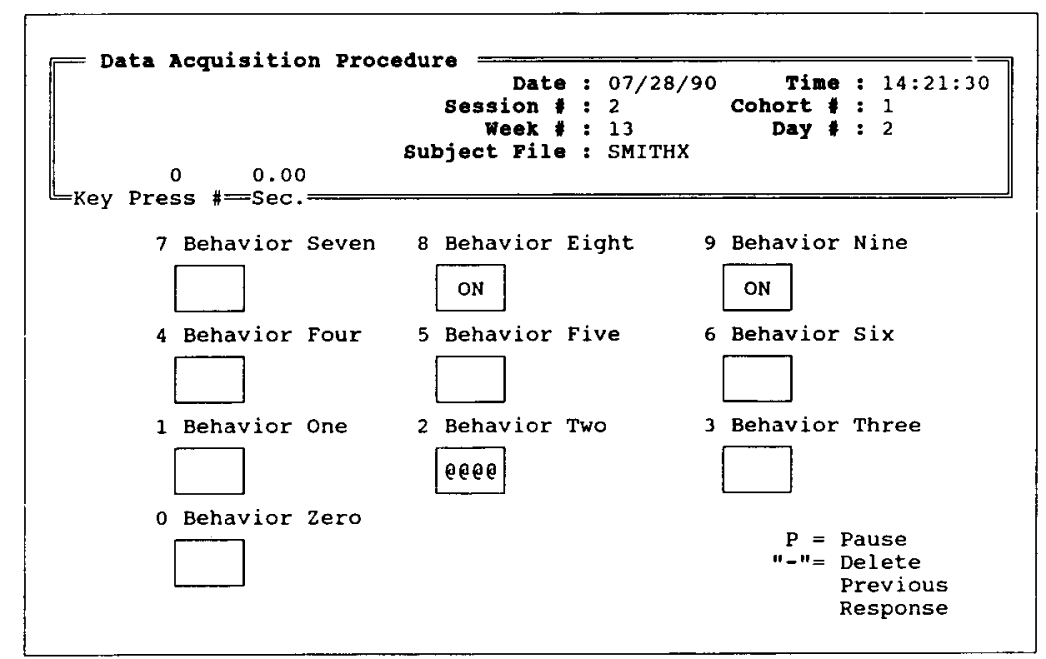

Figure 1. Acquisition mode screen configuration. Labels for the numeric keys are derived from BEHAVS.DBF database file. The subject file, date, and time are user defined. "Cohort, week, and day" are user-defined variable labels. "Key Press \#" represents number of keypresses made for current subject. “@@@@” is a screen marker that appears when the corresponding key is pressed. "ON" indicates behavior timing. 
licenses benefit by having additional resources available through dBASE for manipulating and summarizing data in the database files.

For each subject, there are two output files: "Name".DBF and "Name"'X.DBF (where "Name" represents a subject identifier). Both of these are created automatically in the initialization procedures during the first run of the program for any subject. The amount of data that can be collected and stored is dependent on disk space, but even active observational conditions result in very manageable output files. For example, the two dBASE compatible files that contained $40 \mathrm{~h}$ of observation data for our most active patient (i.e., rate $=116.85$ self-injurious behaviors per hour) were less than $300 \mathrm{~K}$ in size combined.

Keypresses and respective times of occurrence within a session are stored to "Name"'.DBF. Thus, this file contains, for each session observed, only the key number (i.e., 0 to 9) and time, recorded in seconds, from the beginning of the session when a numeric key was pressed. The raw data are stored sequentially to this file as they occur during the scoring mode, and subsequent data for the same subject are appended to this file.

The database file "Name' $X$.DBF contains summary data. For each observational session, summaries of the frequencies and durations of the respective behaviors are compiled. In addition, session descriptors (e.g., date and time), session duration, and ratings scores are stored in this file.

Once the data are stored to disk, subsequent programs may be executed for further analysis. We developed a simple program to summarize several behavioral variables across the 10 weeks of our study of self-injurious behavior. The output includes, for example, descriptive statistics such as averages, sums, and rates of behaviors, observational durations, and sessions. Also, time budget analyses can be easily performed using the behavioral duration data. Other straightforward programs maybe written to analyze temporal features of the raw data (e.g., sequence analysis).

\section{Field Testing}

The program was tested as part of an ongoing observational study investigating the efficacy of Naltrexone in the treatment of self-injurious behavior in developmentally delayed subjects. The study was double-blind and placebo controlled with multiple baselines. Treatment with Naltrexone or placebo occurred on Monday and Wednesday at 8:00 a.m.

Eight subjects were observed 4 days a week for 9 weeks. On Monday, Tuesday, and Thursday, the subjects were nonintrusively videotaped in their natural environments (i.e., bedroom, group lounge, or play area) for six 5-min observation sessions: three in the morning and three in the afternoon. On Wednesday, the subjects were videotaped twice. They were first taped performing a sorting task designed to assess learning and, then, for $10 \mathrm{~min}$ following the task. The videotape analysis method of data collection was chosen for the following reasons: (1) the scoring task may be divided into more manageable segments to combat observer fatigue, (2) in case of scoring difficulties or observer error, the tape may be rescored, and (3) interobserver agreement is readily assessed. In addition, the distractions and logistical challenges of the clinical setting were significant enough so as to impair, if not prohibit, on-site data collection with laptops, even though laptops would have been sufficiently portable for the task.

The videotaped observational sessions were individually scored by 1 of 3 observers for occurrence of selfinjurious behavior and duration of stereotypy and selfrestraint during the session. A total of $102 \mathrm{~h}$ of tape was scored. Table 1 shows the repertoire of behaviors for the 8 subjects. These behaviors were used to create customized behavior codes for each subject and reflect the diversity that can appear even with a few subjects.

To determine interobserver reliability, a 2 nd observer rescored $9.75 \mathrm{~h}$ of randomly selected observational samples across all subjects. Spearman rank correlation coefficients were calculated because the data were not normally distributed (self-injurious behavior: $M=6.09, S D=$ 16.38; stereotypy: $M=50.71, S D=85.53$; self-restraint: $M=62.68, S D=186.60$ ). Interobserver reliability for self-injurious behavior occurrence was $r=0.81$ $(n=94)$. For the duration behaviors, stereotypy and selfrestraint, it was $r=0.89(n=94)$ and $r=0.96(n=94)$, respectively. As shown in Table 2, the range of frequency and duration measures is substantial, reflecting the versatility and reliability of ODAP.

\section{System Requirements and Considerations}

ODAP was written in dBASE III PLUS programming language (Ashton-Tate, 20101 Hamilton Ave., Torrance, CA 90502) and compiled with the Clipper Compiler (Nantucket Corp., 12555 W. Jefferson Blvd., Suite 300, Los Angeles, CA 90066). dBASE software is not necessary to execute ODAP or to browse, edit, or otherwise modify

Table 1

Behavioral Repertoires for SIB Subjects

\begin{tabular}{ll}
\hline \multicolumn{1}{c}{ Behavior } & $N$ \\
\hline Head & 5 \\
Bang & 6 \\
Hit & 3 \\
Slap & 2 \\
Head to shoulder & 2 \\
Pull hair & 1 \\
Poke/hit neck & 1 \\
Torso & \\
Bang on back rest & 2 \\
Arm & 1 \\
Hit & 1 \\
Pinch & \\
Bite & 2 \\
Leg & 2 \\
Hit & \\
Other & \\
\hline
\end{tabular}

Note-SIB $=$ self-injurious behavior. 
Table 2

Subject Characteristics

\begin{tabular}{ccccc} 
Subject & $\begin{array}{c}\text { Frequency } \\
\text { of SIB } \\
\text { (per hour) }\end{array}$ & $\begin{array}{c}\text { Duration of } \\
\text { Stereotypy } \\
(\%)\end{array}$ & $\begin{array}{c}\text { Duration of } \\
\text { Self-Restraint } \\
(\%)\end{array}$ & $\begin{array}{c}\text { Check } \\
\text { Time } \\
(\mathrm{min})\end{array}$ \\
\hline J.V. & 1.07 & 11.00 & - & 85 \\
D.R. & 5.84 & 2.66 & - & 85 \\
P.G. & 14.97 & 7.47 & - & 60 \\
C.R. & 32.35 & 0.00 & - & 100 \\
T.M. & 36.65 & 5.75 & 32.46 & 45 \\
M.C. & 91.94 & 60.69 & - & 60 \\
R.T. & 116.85 & 0.70 & 77.87 & 75 \\
M.K. & 354.50 & 0.46 & - & 45 \\
\hline Note-SIB $=$ self-injurious behavior; $-=$ not applicable.
\end{tabular}

program and output files since these capabilities are available through various menu options within ODAP.

The program must be executed within the MS/PC DOS environment (Version 2.0 or greater) on an IBM XT/AT/PS 2 or compatible and requires a minimum of $317 \mathrm{~K}$ of memory. For most applications, where less than roughly 400 keypresses are made during an observation session, the data are stored in memory and written to disk upon termination of recording. However, when the memory buffer reaches maximum capacity, the data are written to disk, momentarily interrupting recording.

The executable program file (ODAP.EXE) must exist in the same directory as the following database files: "Name"'.DBF; "Name' X.DBF; BEHAVS.DBF, contains all subject codes; RATINGS.DBF, contains userdefined rating prompts; LABELS.DBF, for user-defined variable labels; ODAPDBF.DBF, template for creating "Name"'DBF; and ODAPDBFX.DBF, template for creating "Name' $X$.DBF. These files must be located in the "current"' DOS directory so that they may be searched and/or appended during program operation.

ODAP records data with 100 th of a second timing resolution. This is actually the time at which the software reads the keypress from the keyboard memory and is influenced by internal keyboard nuances that vary among keyboard models. The minimum system requirements include a keyboard (preferably with a numeric key pad), an external floppy drive, and a monitor able to display 80 columns and 24 rows of text. To facilitate program execution, as well as data storage and manipulation, a system operating at $8.0 \mathrm{MHz}$ or faster is recommended.

\section{Discussion}

The use of personal computers and specialized software can greatly ease the difficulties of conducting observational research and enhance data collection procedures. The Observational Data Acquisition Program demonstrated versatility, efficiency, and reliability while collecting real-time counted and timed behavioral data.

The program can be executed from a laptop in the field or from a stationary computer in the lab adjacent to either video equipment or the subject(s) of interest. Although executing ODAP from a laptop does not provide as portable an event recorder as specialized hand-held computers or the previously mentioned bar code scanner, the timing accuracy, screen feedback, and greater data capacity make ODAP and EVENTLOG attractive alternatives. Also, software packages such as these are quite economical in that they utilize and extend the use of common microcomputers available to most researchers, and no specialized hardware is necessary.

An advantage of ODAP was that single keystrokes on a numeric key pad, without carriage returns, facilitated speed, accuracy, and learning. As Flowers and Leger (1982) point out, "the use of single key presses without the carriage return may be more suitable when observing rapidly changing behaviors"' (p. 229). EVENTLOG requires that keys be pressed for the duration of a given behavior such that it might be necessary to press several keys simultaneously. The screen template and current status displayed to the screen during recording and the single keystroke method make ODAP most appropriate for the recording of rapidly changing behaviors. Indeed, the interobserver reliabilities were robust given the diversity of the target behavior rates and durations (Table 2). EVENTLOG, with its capacity to record $40+$ behaviors at a time, appears better suited for recording many slower changing behaviors.

ODAP's database management features make it particularly suitable for large amounts of data. EVENTLOG creates a separate file for each observational session recorded, whereas ODAP appends existing data files. For the data collected during the present field testing, where 8 subjects were observed for multiple 5-min sessions totaling $104 \mathrm{~h}$, EVENTLOG would have created a total of 1,248 data files. ODAP created two files per subject to store raw and summed data (i.e., 16 data files total). In addition, ODAP allowed viewing and editing of database files, as well as generation of ASCII files from the main menu.

In conclusion, ODAP facilitated the conduction of observational research. The single-stroke data entry method, automatic summarization, and data storage to disk minimized personnel training, labor, and human error. The implementation of the software on existing microcomputers was economical and ensured reliable and precisely timed recordings.

\section{Availability}

ODAP is available from Curt A. Sandman, Research Box 5A, 2501 N. Harbor Blvd., Costa Mesa, CA 92626. Requests should be accompanied by a blank, formatted diskette (either 5.25 or $3.5 \mathrm{in}$.).

\section{REFERENCES}

Altmann, J. (1974). Observational study of behavior: Sampling methods. Behaviour, 49, 227-265.

Cone, J. D., \& Foster, S. L. (1982). Direct observation in clinical psychology. In P. C. Kendall \& J. N. Butcher (Eds.), Handbook of research methods in clinical psychology (pp. 311-354). New York: Wiley.

Crossman, E. K., Willams, J. G., \& Chambers, J. H. (1978). Using the PET microcomputer for collecting and analyzing observational data in the classroom. Behavior Research Methods \& Instrumentation, 10, 563-566. 
Eiler, J. M., Nelson, W. W., Jensen, C. C., \& Johnson, S. P. (1989). Automated data collection using bar code. Behavior Research Methods, Instruments, \& Computers, 21, 53-58.

Euis, M. F. (1984). Handheld computers for recording timed behavior observations. Ethology \& Sociobiology, 5, 59-60.

FitzPATRICK, L. J. (1977). Automated data collection for observed events. Behavior Research Methods \& Instrumentation, 9, 447-451.

Flowers, J. H., \& Leger, D. W. (1982). Personal computers and behavior observation: An introduction. Behavior Research Methods \& Instrumentation, 14, 227-230.

HAwkINS, R. P. (1982). Developing a behavior code. In D. P. Hartmann (Ed.), Using observers to study behavior: New directions for methodology of social and behavioral science (pp. 21-35). San Francisco: Jossey-Bass.

KoONTZ, F. W. (1982). WATCH: Microcomputer programs to collect and analyze behavioral observations based on focal-animal sampling. Behavior Research Methods \& Instrumentation, 14, 431-432.
LEHNER, P. N. (1979). Handbook of ethological methods. New York: Garland STPM Press.

Lifshitz, K., O'Keeffe, R. T., LeE, K. L., Avery, J. (1985). KIBOS: A microcomputerized system for the continuous collection and analysis of behavioral data. Applied Animal Behaviour Science, 13, 295-218.

SACKett, G. P., Stephenson, E., Ruppenthal, G. C. (1973). Digital data acquisition systems for observing behavior in laboratory and field settings. Behavior Research Methods \& Instrumentation, 5, 344-348.

SIDOwskı, J. B. (1977). Observational research: Some instrumented systems for scoring and storing behavioral data. Behavior Research Methods \& Instrumentation, 9, 403-404.

(Manuscript received March 28, 1990; revision accepted for publication December 4, 1990.) 